\title{
Cadaveri di pietra \\ Tiziano Terzani dinanzi al crollo del comunismo
}

Mauro Novelli

DOI: http://dx.doi.org/10.7359/826-2017-nove

\section{Abstract}

In 1991, while traveling by boat along the Amur River, on the border between the USSR and China, Tiziano Terzani was surprised at the news of the coup against Gorbachev in Moscow. He decided to document the collapse of the Soviet power in Asia, moving on to Siberia, Turkestan, Caucasus, and finally Moscow. It is a journey through the ruins of a dream, from which came a bitter book, Goodnight, Mr. Lenin. Begun as a travel story, the book turns into a thriller. How and why communism died? Who is the killer? Where is the body? The fate of the statues of Lenin, then ubiquitous, helps to answer these questions. The contribution focuses on representative methods and the interpretation of this monumental heritage.

Parole chiave: Asia sovietica, comunismo, Lenin, statua, Tiziano Terzani.

Keywords: communism, Lenin, Soviet Asia, statue, Tiziano Terzani.

L'uscita nel 1992 di un libro come Buonanotte, signor Lenin ${ }^{1}$ poté entusiasmare, ma non sorprendere i tanti affezionati lettori di Tiziano Terzani. Una volta di più il giornalista fiorentino rinnovava il suo corpo a corpo con il comunismo asiatico, aggirandosi desolato fra le macerie di un sogno

1 Ora in Terzani 2011, 969-1407. Mancano da questa edizione (dalla quale saranno tratte le citazioni nel testo, con rimando al numero di pagina) le 47 fotografie scattate dall'autore durante il viaggio, presenti nella princeps (Terzani 1992) e in varie ristampe, dove il titolo dell'opera conosce oscillazioni. Nel tascabile TEA (2006 $)$, ad esempio, al Buonanotte signor Lenin della copertina fa riscontro il Buonanotte, Signor Lenin del frontespizio, a sua volta contraddetto nella pagina successiva dal Buonanotte, signor Lenin dell'elenco di opere di Terzani presenti nella collana. 
scaduto a incubo. L'idea di partenza però era un'altra: raccontare una spedizione in nave lungo il fiume Amur, che per un lungo tratto segnava il confine fra URSS e Cina, in territori remoti, fascinosi e carichi di storia, vietati a viaggiatori e cronisti da decenni.

Dietro la patina vintage, si riconosce nell'impresa il crescente disagio dell'autore nei confronti dei ritmi ansiosi della società occidentale. Nei libri successivi, a partire da Un indovino mi disse (Terzani 1995), il rallentamento diviene addirittura il tema portante. La rapidità, tanto decisiva nel mestiere del reporter, cede il passo all'esigenza di un confronto più riposato con le vestigia di civiltà millenarie, travolte dal socialismo reale. Nel contempo aumentano gli spazi concessi ai ragionamenti, alle digressioni, ai flashback in cui si modella con nettezza il profilo di un uomo al quale i panni del reporter vanno sempre più stretti.

Il desiderio di adeguarsi al battito dell'Asia profonda del resto già emerge nella reazione al golpe contro Gorbačëv, che Terzani apprende da una radiolina sul ponte della nave. Al riflesso immediato, che lo porta a cercare febbrilmente di procurarsi un biglietto aereo per Mosca, succede una decisione originale: non intrupparsi fra gli inviati in rotta verso la capitale ma attraversare in perfetta solitudine le repubbliche sovietiche, per documentare in diretta il crollo dell'Unione negli sterminati possedimenti orientali. Il viaggio dura due mesi abbondanti: ai primi di ottobre del 1991 Terzani raggiunge finalmente Mosca, dopo avere attraversato la Siberia, il Turkestan e il Caucaso saltando da un aereo traballante a un taxi improvvisato, schivando le insidie di spie e ras locali, mandando fra mille impedimenti i suoi pezzi al Corriere della Sera. La valorizzazione delle modalità con cui riesce ad agguantare una notizia, o a visitare un luogo in circostanze rocambolesche è il sale della maniera di Terzani (Novelli 2014), che una volta di più documenta il fallimento di una rivoluzione.

Si ha l'impressione, scorrendo il primo dei due "Meridiani" Mondadori riservato alle sue opere, che per decenni non abbia fatto altro che aggirarsi fra le rovine del medesimo sogno. Non a caso il libro è dedicato alla memoria del padre, che nelle idee comuniste, come milioni di altri italiani, aveva creduto. La coscienza di questa illusione rende più amari i sopralluoghi. Terzani constata di persona i disastri ecologici, la miseria dilagante, la bruttezza degli edifici, la rassegnata incuria verso il lavoro là dove si era proclamata l'emancipazione dei lavoratori. Coglie, dopo decenni di russificazione, il prepotente risorgere dalle ceneri dei nazionalismi e dell'astio fra popoli vicini. I burocrati del vecchio regime, rinfoderato il rosso e cambiato nome al partito, restano saldi dietro le loro scrivanie. Ma nell'ombra si lavora a sostituire falci e martelli con la 
mezzaluna islamica, mentre le mafie prosperano sfruttando corruzione e vuoti legislativi.

Uscito nel settembre del 1992, quando da poco il PCI fra indicibili travagli si era trasformato in Partito Democratico della Sinistra, Buonanotte, signor Lenin era destinato a suscitare polemiche in Italia, dove l'autore fu scambiato con uno dei tanti sciacalli pronti a sfruttare l'occasione propizia per avventarsi sulle spoglie del comunismo. Un abbaglio, certo. Nel quale dovette giocare un ruolo l'enfasi posta da Terzani sulla metafora in questione. Partito come un racconto di viaggio vecchio stile, adagiato sui ritmi lenti e sinuosi di un fiume siberiano, il libro finisce col trasformarsi in una sorta di thriller, e il narratore in un detective che di continuo torna a porsi le medesime domande. Come e perché è morto il comunismo? Si è trattato di un decesso per cause naturali o di un delitto? In questo caso, chi è l'assassino? E ancora, e soprattutto: dove sta il cadavere?

È una vecchia ossessione, rintracciabile sin dalle prime righe del libro d'esordio, Pelle di leopardo (Terzani 1973), dove campeggia l'immagine di un corpo rigido, "rovesciato sull'argine di un campo con le braccia aperte, le mani magrissime piene di fango e la faccia gialla, di cera, con gli occhi vuoti a guardare il cielo" (Terzani 2011, 79). Vedere il morto da vicino, il più vicino possibile. Sia l'anonimo vietnamita straziato, o il Grande Timoniere. La Cina dei primi anni Ottanta, nella prefazione alla ristampa 1998 della Porta proibita (Terzani 1984), è paragonata a "una sorta di enorme zattera alla deriva appesantita dalla zavorra di un cadavere" (ibid., 643). Quello che s'incontra incominciando la lettura:

Al centro del centro della Cina c'è un cadavere che nessuno ha il coraggio di portar via. Avvolto nella bandiera rossa del partito, protetto in una bara di vetro, dentro un enorme mausoleo in mezzo alla piazza della Pace Celeste, il corpo imbalsamato di Mao rappresenta il legame simbolico della Cina di oggi col suo passato, ma anche il punto di riferimento per il suo futuro. (ibid., 653)

Buonanotte, signor Lenin finisce là dove La porta proibita inizia. Dalla visione di un corpo artificialmente sottratto alla decomposizione, assurto a emblema di un'ideologia che "ha esaurito la sua creatività, ha perso la sua abilità di ispirare e di commuovere" (ibid., 662). Moltiplicata per mille, la sentenza si estende ai simulacri di pietra, marmo, bronzo o cemento dei due leader, disseminati in ogni dove ma incapaci di parlare al popolo miserabile che li attornia. Nell'oasi di Kashgar, dove vivono gli uiguri, "Di fango son le case, di fango son le strade, le moschee, le tombe. / Solo 
Mao è di granito" (ibid., 916). La sua statua, "Torreggiante sul labirinto medievale di questa città d'ocra brulicante di gente dall'aspetto antico, di pecore, muli e cammelli", appare a Terzani "bizzarra, sproporzionata, fuori posto" (ibid.).

È già in nuce, qui, il motivo che attraversa l'esplorazione dell'Unione Sovietica, dove Lenin era solito presidiare ogni villaggio, sotto specie di busto modesto o di statua ciclopica, "sempre li, dovunque presente con il berretto o il giornale in una mano e l'altra alzata per indicare il futuro o semplicemente l'orizzonte vuoto" (ibid., 976). Almeno sino al 1991. In seguito il cinema si è incaricato di dare rappresentazioni esemplari dello smaltimento di un simbolo ormai scomodo. Vedi la chiatta lentissima che ne trasporta un esemplare sezionato lungo il Danubio, nel film di Theo Angelopoulos Lo sguardo di Ulisse (1995); oppure l'elicottero che in Good Bye, Lenin (2003), di Wolfgang Becker, lo fa sparire dagli occhi dei berlinesi.

È il tramonto di un'epoca quello che Terzani vive in diretta, restando sorpreso dal mancato esplodere della violenza, a valle di rancori lungamente covati. Non sono più i tempi di Saigon. Tutto si riduce a una resa dei conti con le statue. Abbatterle o lasciarle in piedi? "I comunisti non ci sono più, è vero, almeno in apparenza, ma forse è altrettanto vero che non ci sono mai stati e che la cosiddetta fine del comunismo ora - almeno nell'Asia centrale - non è nient'altro che la fine di un regime coloniale" (Terzani 2011, 1159). Lo schiacciante dominio di un'ideologia importata sulla vita vissuta si rispecchia nel contrasto fra l'altera immobilità dei monumenti e il brulicante andirivieni della realtà circostante. Gli unici uomini felici incontrati durante il viaggio sono quelli scolpiti, "patetico tentativo di compensare con un mondo di fantasia la miseria del mondo reale; un modo per dare almeno una immagine di quel che non è stato possibile. Il socialismo è nato pieno di sogni. Non li ha realizzati, ma li ha almeno messi nei bronzi e nelle pietre" (ibid., 1406).

Il mito della statua animata svanisce nell'ombra. Non è la pietra che si fa carne, ma la carne che si fa pietra, senza remissione. I fantasmi del passato continuano a marcare immobili un territorio che non è mai appartenuto loro sino in fondo. Il paragone con la natia Firenze sorge inevitabile. Discendente da una famiglia di scalpellini, Terzani imparò presto a misurare l'impatto dei monumenti sulla psiche. Il ricordo delle strade percorse nelle mattine d'infanzia, all'ombra di palazzi, statue e chiese, si incide nel profondo dell'identità. Da adulto, ogni volta che il treno, avvicinandosi alla stazione di Santa Maria Novella, gli restituisce la cupola del Duomo e la cima del campanile di Giotto, è un tuffo al cuore. Ma cosa proverà chi 
ritorna in una desolata città della Siberia, e uscito dall'aeroporto non vede altro che "casacce di mattoni grigi, pali della luce in cemento, macchine parcheggiate alla rinfusa” (ibid., 1097), sporcizia e squallore?

I monumenti concretizzano visivamente lo scarto fra un ideale e i suoi rottami. Così sul lungofiume di Komsomol, agglomerato costruito dai prigionieri del gulag, fra bottiglie rotte e calcinacci svetta il monumento alla gloriosa avanzata del progresso. Intellettuali, soldati, ingegneri, risolute pioniere sono pronti a marciare verso un'alba divenuta tramonto. Ad Habarovsk, a Blagoveščensk, negli altri centri dell'Estremo Oriente sovietico Lenin continua a indicare la retta via, mentre la tv nei caffè fumosi reca le notizie delle dimostrazioni pro-Eltsin. Ai piedi del vecchio rivoluzionario, una mano anonima ha deposto un mazzo di rose rosse, fresche. Ma è difficile distinguere l'omaggio spontaneo "da quel che è programmato per parere spontaneo. La produzione di trompe-l'oeil è stata una delle specialità del comunismo" (ibid., 1016).

Le stesse riflessioni sono suscitate dai monumenti intravisti in tante città dell'Asia, durante le corse mattutine per tenersi in forma e osservare la vita quotidiana senza dare troppo nell'occhio. Quanto potrà resistere, ad Alma Ata, il monumento al fondatore del KGB Dzeržinskij, incappottato nei pressi della stazione centrale di polizia? Il locale museo è già in fase di ristrutturazione: il custode dovrà imparare l'ennesima versione della storia, piegata alle esigenze del nuovo potere. Ad Osh, in Kirghisia, Terzani chiede ai nuovi potenti se pensino di sostituire Lenin con Madamin Beck, il capo dei ribelli basmacci, che dopo la Grande Guerra diedero parecchio filo da torcere all'Armata Rossa. Si sente rispondere che è presto. Ad oggi la mastodontica statua resta ancora al suo posto, risparmiata grazie anche agli stretti legami del Paese con la Russia. Ma è ormai ridotta a un relitto, sopravvissuto ai valori che impersonava. All'inverso gli antichi monumenti islamici dell'Asia centrale sono tornati a pulsare, mentre nel 1991, dopo decenni di comunismo, apparivano gusci inanimati: "Le grandi moschee di Samarcanda dalle cupole color turchese, le famose medressa, le scuole coraniche dalle facciate ricoperte di mosaici, ci sono ancora, in parte anche restaurate, ma sono come cadaveri imbalsamati cui non resta più un filo della vita d'un tempo" (ibid., 1202).

In altri casi i nuovi regimi si sono preoccupati di abbattere i monumenti eretti dai sovietici. Come ha osservato Katherine Verdery, "desecrating a statue partakes of the larger history of iconoclasm. Tearing it down not only removes that specific body from the landscape, as if to excise it from history, but also proves that because it can be torn down, no god protects it" (Verdery 1999, 5). Nel parco di Tashkent non esiste più 
la "bella testa in bronzo di Karl Marx in cima a una colonna di granito. Con la sua fronte bombata e gli zigomi alti, sembrava il ritratto di uno dei tanti vecchi venditori del bazar" (Terzani 2011, 1198). In suo luogo nel 1999 è sorto un monumento a Tamerlano, reinventato eroe nazionale dal presidente Islam Karimov. A Dušanbe, capitale del Tagikistan, un emiro persiano del IX secolo, Isma'il ibn Ahmad, ha sostituito Lenin, la prima statua a cadere nell'Asia sovietica, abbattuta da una folla di contadini all'alba del 22 settembre 1991.

Terzani, fra i pochi occidentali presenti in città, ebbe la ventura di assistere. "Gli hanno buttato una corda d'acciaio al collo, una grossa gru gialla si è messa a tirare, e Lenin, lento, come se non volesse lasciare quel piedistallo sul quale troneggiava da settant'anni, s'è piegato da una parte e s'è accasciato in frantumi" (ibid., 1258). È una delle pagine più intense del libro, dove emerge con maggior nettezza l'antitesi fra i comunisti, scettici, disillusi, stretti nei loro scialbi abiti moderni, e i musulmani giunti dalle campagne. "'Svegliati, popolo', intonava il mullah e la folla ripeteva: 'Popolo, svegliati, svegliati'. Era strano sentire queste parole dinanzi al Lenin condannato a morte da un popolo che lui aveva voluto svegliare e che ora accusava lui di averlo addormentato" (ibid., 1261). Il comunismo oppio dei popoli. Terzani è testimone tempestivo del ritorno alla ribalta dell'Islam, tenacemente sopravvissuto nell'ombra per decenni:

Il tonfo di quella massa di bronzo che cadeva sul marmo rosa del podio nessuno l'ha sentito, subissato com'era da un urlo antico che improvvisamente, come riaffiorato dalle viscere della terra, ha fatto tremare la piazza e, simbolicamente, tutta questa immensa regione di montagne e deserti nel cuore dell'Asia: "Allah... Allah... Allah Akbar", Allah è grande.

La più colorata e straordinaria folla che uno possa immaginarsi, tutta di uomini, era in piedi, in estasi, con migliaia di pugni e mani alzati, come ad aiutare quella caduta: vecchi dalle lunghe barbe bianchissime e i caftani a strisce gialle, verdi e rosse; giovani in tabarri violetti e azzurri; mullab dalle tuniche marrone e grigie, tutti con in testa il loro zucchetto nero, quadrangolare, con fregi in bianco. (ibid., 1261)

Di lì a poco la moltitudine può gettarsi a capofitto sulla carcassa del padre della Rivoluzione, subissato nella polvere di calci e sputi. È una delle rarissime scene di violenza rintracciabili in Buonanotte, signor Lenin. Le altre repubbliche asiatiche dilazionano il problema, oppure agiscono con discrezione. In Armenia ad esempio Terzani si imbatte nel corpo bronzeo di Lenin disteso nel cortile di un museo. Nessuno sa dove sia andata a finire la testa. In Turkmenia il governo locale fa sparire nottetempo la statua del rivoluzionario russo dalla via maestra della capitale Ašhabad. Al mattino 
sul piedestallo di marmo non rimane nulla. La gente simula indifferenza e finge di credere alla versione ufficiale: gli operai l'hanno rimossa per ordine del partito, in quanto la scarsa qualità artistica ne rendeva inappropriata la presenza nel centro cittadino. Qualche anno più tardi la Turkmenia tornerà a popolarsi di enormi statue, questa volta dorate, raffiguranti Saparmurat Nyazov, il Türkmenbaşy (padre di tutti i turkmeni), padrone assoluto del Paese, nel quale imporrà la presenza ossessiva della sua effigie, condita con bizzarrie megalomani abrogate solo dopo la sua morte, nel 2006.

Sic transit gloria mundi. "I monumenti sorpassati dalla storia hanno un loro speciale modo di essere ironici” (Terzani 2011, 1375), si tratti del grande mazzo arrugginito di fiori metallici, che sul confine vorrebbe celebrare "l'amicizia indistruttibile del popolo georgiano, armeno e azero", del monumento di Baku alla leggendaria spia comunista Richard Sorge, o della Cascade di Erevan, scenografica scalinata in marmo iniziata nel 1971 come celebrazione del potere sovietico. Terzani vi incontra casualmente il responsabile della costruzione, Jim Torosyan, che la contrabbanda come glorificazione dell'Armenia, della "forza della vita che trionfa su tutto", del "cammino di un popolo" (ibid., 1390).

Esito obbligato dell'itinerario fra le rovine del sogno collettivista non può che essere Mosca. Nella Piazza Rossa Terzani vede ancora sventolare la bandiera sovietica, che verrà ammainata a Natale. È l'occasione per ragionare sullo scarto fra le nobili aspirazioni del comunismo, e il sistema inventato dai suoi sacerdoti. La ricerca dell'assassino si salda alla visione del cadavere. Il corpo di Lenin stagiona in un mausoleo a forma di piramide. È un paradosso che non smette di sconcertare. L'uomo che aveva invitato i russi a scoperchiare le bare dei santi, per verificare come il miracolo della conservazione dei corpi fosse una leggenda, giace dagli anni Venti in una teca di vetro, esposto alla curiosità dei visitatori, ridotto a mummia periodicamente sottoposta a manutenzione per tutelarne le parvenze umane. Terzani in ultimo si dice convinto che la rimozione sia imminente, poiché ogni nuovo sistema, nell'instaurarsi, deve cancellare o per lo meno riformulare la memoria dei predecessori. Eppure, "Se la rivoluzione che ha luogo ora in questo immenso paese volesse davvero fare qualche cosa di nuovo potrebbe impegnarsi a non distruggere i monumenti del socialismo. Servirebbero, se non altro, a ricordare quali erano le sue aspirazioni” (ibid., 1406).

Così è stato, sinora, per la mummia di Lenin come per le statue, stele, busti dove sopravvive la sua figura, conservata ora dalla nostalgia ora dal timore, ora dalla pigrizia ora dalla noncuranza. Nel XXI secolo Vladimir Il'ič Ul' janov continua a scrutare l'orizzonte dall'Artico all'Antartico, dal- 
la ghost town mineraria di Pyramiden alla stazione meteorologica abbandonata della Terra di Enderby. A Saratov, sul Volga, le navi che si affacciano sulla tratta vedono stagliarsi il profilo del leader bolscevico, alto ben 27 metri; a Ulan Ude, sulla Transiberiana, i passeggeri scorgono dai finestrini una sua enorme testa. Migliaia di repliche punteggiano tuttora il territorio dell'ex URSS (Gessen 2016) compresa l'Ucraina, sebbene parecchi esemplari siano stati atterrati o vandalizzati in seguito al conflitto con la Russia. Né sono mancate reinterpretazioni beffarde: l'artista Alexander Milov è arrivato a trasformare il Lenin di Odessa in un minaccioso Darth Vader (Bakare 2015).

Altrove, Lenin non sopravvive soltanto nei Paesi rimasti fedeli ai suoi ideali. Nella nazione del blocco occidentale che per decenni ha avuto il partito comunista più forte, l'Italia, si registrano un paio di presenze, entrambe a loro modo significative. A Capri, nei bellissimi Giardini di Augusto, una stele realizzata da Giacomo Manzù nel 1970 ricorda il duplice soggiorno del rivoluzionario, che fu ospite di Maksim Gorkij nel 1908 e 1910. A Cavriago, borgo nella pianura reggiana, si può ammirare nei pressi di un parcheggio, dirimpetto a villette di nuova costruzione, la copia di un busto fuso in Ucraina nel 1922: uno dei pochissimi realizzati prima della morte del leader. Giunto nella penisola per vie traverse nel secondo dopoguerra, nel 1970 il busto venne messo a dimora nel comune dove nel '19 gli operai avevano proclamato la volontà di seguire il luminoso esempio della Rivoluzione d'Ottobre. La mozione, ripresa dall'Avanti!, ebbe la ventura di essere citata durante il primo congresso della Terza Internazionale dallo stesso Lenin, che la portò a esempio del progredire del comunismo nel mondo: "[...] possiamo dire a buon diritto che le masse italiane sono per noi, che le masse italiane hanno capito che cosa sono i sovietisti russi" $^{2}$. Negli stessi giorni, un poco più a nord, a Milano, Benito Mussolini fondava i Fasci di combattimento.

\section{RIFERIMENTI BIBLIOGRAFICI}

Bakare, Lanre. 2015. "The Force Awakens (in Ukraine): Darth Vader Statue Replaces Lenin Monument”. The Guardian, October 23.

Caliceti, Giuseppe. 2004. Il busto di Lenin. Milano: Sironi.

2 Il brano è tratto dall'epigrafe a Caliceti 2004, romanzo ambientato nei mesi della dissoluzione del PCI a Cavriago, dove un gruppo di irriducibili pensionati si stringe intorno al busto di Lenin. 
Gessen, Masha. 2016. “The Soviet Union Is Gone: Why Do Lenin Statues Remain?”. The New York Times, June 14.

Novelli, Mauro. 2014. “Tiziano Terzani. Stile e tecniche narrative”. In Tiziano Terzani. Guardare i fiori da un cavallo in corsa, a cura di Àlen Loreti, 97-101. Milano: Rizzoli.

Terzani, Tiziano. 1973. Pelle di leopardo. Diario vietnamita di un corrispondente di guerra 1972-1973. Milano: Feltrinelli.

Terzani, Tiziano. 1984. La porta proibita. Milano: Longanesi.

Terzani, Tiziano. 1992. Buonanotte, signor Lenin. Milano: Longanesi.

Terzani, Tiziano. 1995. Un indovino mi disse. Milano: Longanesi.

Terzani, Tiziano. 2011. Tutte le opere, vol. I, a cura di Àlen Loreti. Milano: Mondadori.

Verdery, Katherine. 1999. The Political Lives of Dead Bodies: Reburial and Postsocialist Change. New York: Columbia University Press. 
